GÉNERO E IDENTIDAD

\title{
APORTE DE LA MUJER RAMA AL DESARROLLO IDENTITARIO DE LAS COMUNIDADES DE RAMA CAY Y LA ZOMPOPERA, BLUEFIELDS
}

\author{
María Luisa Morales ${ }^{[15]}$ \\ Martina Thomas McCrea ${ }^{[16]}$ \\ Neidy Gutiérrez Soza ${ }^{[17]}$
}

\section{Resumen}

Históricamente el pueblo rama es el grupo indígena de la Costa Atlántica de menor población. El pueblo rama tiene una notable variedad de recursos naturales, entre ellos el recurso forestal y la pesca.

Este estudio contribuye a un acercamiento sobre la realidad socioeconómica y cultural del pueblo rama, desde las voces de las mujeres y particularmente visibilizar el aporte de ellas, orientado al mejoramiento de las condiciones de vida de las familias y por ende de la comunidad a través del fortalecimiento de su identidad y cosmovisión.

El estudio se desarrolló en las comunidades de Rama Cay y la Zompopera. En el ámbito social la mujer rama aporta significativamente a las actividades de organización comunitaria, donde en los últimos años ha manifestado mayor participación y liderazgo. Su labor en la organización comunitaria, también le ha permitido posicionarse mejor en su entorno sociocultural.

En relación a la educación, la mujer rama es quien custodia los valores y las tradiciones, por tanto desde la intimidad de su casa transmite en esencia la cultura rama. En lo que respecta a la dinámica económica, la mujer es la que siembra, cosecha y también comercializa todos los productos tanto de la agricultura como de la pesca.

[15] Licda. en Sociología con Mención en Autonomía, URACCAN-Bluefields.

[16] Licda. en Sociología con Mención en Autonomía, URACCAN-Bluefields.

[17] Máster en Antropología Social. Coordinadora Investigación y Posgrado URACCAN Bluefields.

82 | CIENCIA E INTERCULTURALIDAD, Volumen 4, Año 2, No. 2, Junio 2009 


\section{Introducción}

La Costa Caribe de Nicaragua es multiétnica, multilingüe y pluricultural: tres condiciones que caracterizan su población, compuesta por miskitos, sumos, creoles, mestizos, ramas y garífunas. Los ramas es uno de los Pueblos Indígenas en Nicaragua, cuya población e idioma dramáticamente se está extinguiendo. En la actualidad apenas existen unos diez ancianos hablantes de lengua rama y una población de aproximadamente 1400 habitantes (Mueller, 2003).

El territorio Rama está constituido por la pequeña isla de Rama Cay, ubicada a unos 15 kilómetros al sur de Bluefields, y las comunidades La Zompopera, El Coco, Wiring Cay, Punta de Águila, río Maíz y río Indio.

La población rama es uno de los pueblos indígenas de la Costa Caribe, con la menor cantidad porcentual de la población regional (aproximadamente $0.55 \%$ ) y ubicada en la Región Autónoma Atlántico Sur -RAAS-. El territorio rama administrativamente se encuentra en el municipio de Bluefields.

El pueblo rama tiene una notable variedad de recursos naturales, entre ellos el recurso forestal forma parte importante en sus actividades de desarrollo ya que dependen del bosque y lo utilizan dándole múltiples usos como alimento para consumo humano, medicina y en menor grado como fuente de ingreso. La mayoría de la población está concentrada en la isla de Rama Cay. Está compuesta por dos pequeñas islas unidas por un banco formado por conchas de ostiones y caracoles (Moreno et al., 2000).

Con el presente estudio se busca contribuir de forma general a un acercamiento y entendimiento sobre la realidad socioeconómica y cultural del pueblo rama, desde las voces de las mujeres y particularmente visibilizar el aporte de la mujer orientado al mejoramiento de las condiciones de vida de las familias y por ende de la comunidad en su conjunto a través del fortalecimiento de su identidad y cosmovisión.

El estudio se desarrolló en las comunidades, Isla de Rama Cay por ser la principal comunidad en donde se concentra la mayor población de rama. Por otro lado, una de las razones principales al seleccionar la comunidad de la Zompopera, consistió en la experiencia de trabajo desarrollada con AMIR (Asociación de Mujeres Indígenas Ramas).

Es esta investigación es de interés contribuir a la sensibilización de las autoridades locales, regionales, nacionales e internacionales, sobre la necesidad emergente de trabajar de manera conjunta (de la mano con las mujeres), proyectos de desarrollo 


\section{GÉNERO E IDENTIDAD}

comunitarios que contribuyan a fortalecer sus propias capacidades e incrementen sus conocimientos, para generar cambios significativos en su comunidad, respetando sus tradiciones y costumbres ancestrales.

Sin temor alguno, los resultados de este estudio pueden ser tomados en cuenta como referencia a la hora de diseñar proyectos o acciones de desarrollo por parte del gobierno u otras instancias en cualquier comunidad del territorio Rama.

\section{Revisión de literatura}

Cuando se hace referencia a la situación socioeconómica de las comunidades indígenas de Rama Cay y Zompopera, nos referimos a la situación en cuanto a cobertura de los servicios básicos (salud, educación, vivienda), y a la ausencia de infraestructura social. Así mismo a ser sujetos de financiamiento u otros beneficios para desarrollar actividades productivas que les permitan mejor sus condiciones de vida.

Ante esta situación, Cunnigham (1999), plantea que: "Es indispensable la recomposición de los tejidos sociales, de las relaciones sociales basadas en la cooperación, colaboración, complementariedad desde los niveles locales hasta el ámbito internacional. Afirmando que la recreación cultural emerge precisamente de la capacidad de esa inmensa red humana de potenciar desde el poder local y, a partir de su peso, movilizar los poderes en los otros ámbitos".

Igualmente afirma que la recuperación de la cultura de sostenibilidad, es la base de la economía indígena para fortalecer nuestras capacidades, asegurar la subsistencia y alimentación, así como las oportunidades de participar en las economías de mercado, mediante diferentes mecanismos que partan de los más simples y sencillos y que de forma progresiva se reconfiguren en función del desarrollo desde la propia visión indígena (Cunnigham; 1999).

En ese sentido el trabajo que se presenta contribuye significativamente al debate sobre la necesidad emergente de que los pueblos indígenas puedan alcanzar su propio desarrollo sin tener que despojarse de su propia identidad, vista desde el aporte que la mujer indígena rama hace a la familia y a la comunidad.

\section{Revitalización cultural}

La revitalización cultural debe de entenderse como un proceso en donde las prácticas tradicionales de los pueblos en su máxima expresión, tratan de visibilizarse. Por tanto, 
no se hace referencias al rescate, puesto que no se ha perdido el idioma en algunos pueblos y comunidades étnicas (Obando et al., 1999).

\section{Desarrollo con identidad}

En el pasado, los esfuerzos por mejorar la situación de los pueblos indígenas solían basarse en la idea de que, para beneficiarse con el desarrollo, dichos pueblos debían sacrificar su cultura e identidad y asimilarse o integrarse en la economía y la sociedad nacional. Además, el enfoque aplicado para lograr este objetivo solía ser paternalista y creaba dependencias hacia los gobiernos, las instituciones religiosas o las ONG.

Sin embargo, la experiencia recogida en el BID con algunos proyectos de desarrollo de pequeña escala en ejecución desde hace varios años, y una nueva generación de proyectos de desarrollo comunitario integral y participativo aprobados en los últimos años, han demostrado que el fortalecimiento de la identidad cultural y la promoción del desarrollo socioeconómico sostenible son objetivos que se refuerzan mutuamente en lugar de ser mutuamente excluyentes. Por tanto, cuando los esfuerzos de desarrollo se basan en los valores locales, en las aspiraciones y en la organización social, la cultura se convierte en un activo en vez de en un impedimento para el desarrollo, de tal manera, la población adopta más fácilmente los cambios que los sacarán de la pobreza material (Cárdenas, V, 1997).

Por lo anterior, desarrollo con identidad corresponde al principio de que la cultura no es un obstáculo para el desarrollo, según afirmaba la opinión prevaleciente durante muchos años, sino más bien es el capital inicial para el progreso social y económico sostenible, porque se asienta en los valores, las aspiraciones y el potencial de los pueblos. Ello contrasta con la imposición de un modelo de desarrollo de arriba hacia abajo y desde afuera hacia adentro. Por lo tanto, el desarrollo y la identidad cultural no se excluyen mutuamente, sino que son parte del mismo círculo virtuoso de desarrollo sostenible y adecuado desde el punto de vista sociocultural (Cárdenas; 1997).

\section{Aporte socioeconómico y cultural de la mujer}

Históricamente, las mujeres han desempeñado múltiples roles ante la sociedad. Estos roles se han determinado por medio de la participación, en diferentes espacio y ámbitos, tanto en funciones productivas como reproductivas, comunitarias y culturales, con el fin de visualizar su trabajo.

En consecuencia, las mujeres, según López (1995), desarrollan funciones productivas que contribuyen económicamente al hogar y a la comunidad, estas pueden ser por 


\section{GÉNERO E IDENTIDAD}

ejemplo, agricultura de subsistencia, cultivos de patios, artesanías, entre otros. Por otro lado, la mujer históricamente ha concentrado su papel al cuidado y el mantenimiento del hogar, gestar, dar a luz, criar y educar a los hijos, atención a la salud, preparación de los alimentos, recolección de agua, leña, etc.; estas actividades, lamentablemente se consideran no económicas, por lo que no se tiene una compensación monetaria y por lo general se excluyen de las cuentas nacionales de ingresos.

También las actividades comunitarias y culturales incluyen la organización colectiva de eventos sociales y servicio comunitario entre los que se destacan: ceremonias y celebraciones, actividades para el mejoramiento de la comunidad, participación en grupos y organizaciones, en actividades de la política local y de otra índole. Así mismo, históricamente a las mujeres provenientes de los pueblos indígenas también se les ha asignado el rol protagónico de conservar y sobre todo transmitir las costumbres y tradiciones, siendo esto un rol más dentro de la labor de las mujeres. Por tanto, eso implica, una considerable cantidad de tiempo voluntario y es importante para el desarrollo cultural y espiritual de las comunidades siendo además un vehículo para la autodeterminación (López, 1995).

\section{Economía indígena}

Las diferencias entre la propiedad colectiva y privada para los pueblos indígenas y la tierra, "Sus usos y aprovechamiento" se organizan en torno a sus tradiciones y costumbres ancestrales, estableciendo de este modo un vínculo de sostenibilidad y espiritualidad con respecto a la organización material de la vida y la economía indígena. Por tanto, la unidad del sistema productivo indígena, es sin duda la familia, las cuales están organizadas las actividades tradicionales para la subsistencia con una fuerte división de trabajo por roles de género y con un concepto de colectividad de la tierra (Guglielmi, G, 2006).

Las tierras comunales que no se destinan al uso agrícola no están bien delimitadas y se vuelven vulnerables y susceptibles a la invasión. Los sistemas de producción según Sahlins, establece que en las sociedades primitivas la economía es vista como el proceso de abastecimiento de la sociedad. "La economía se concibe como un componente de la cultura, más que como una particular acción humano. Es el proceso vital y material de la sociedad y no es un comportamiento individual para la satisfacción de las necesidades. Así mismo, el concepto de economía indígena no se corresponde con el concepto capitalista del mismo. Por tanto, son diferentes las motivaciones hacia el esfuerzo productivo y también las metas y finalidades productivas. Para el indígena el trabajo se articula en torno a una estructura y organización sociocultural que pone en 
primer plano la solidaridad y redistribución. Por tanto, es más equitativo que en las sociedades "modernas", donde el estimulo es el interés personal (Guglielmi, G, 2006).

\section{Identidad}

Los Pueblos Indígenas, comunidades étnicas y afrodescendientes de Nicaragua, de acuerdo a Rizo (2003), tienen diferentes grados de mestizajes (cruce biológico), pero esta variable genética no es la que define la identidad étnica en forma absoluta. Por tanto, la identidad étnica es el resultado de una auto adscripción o representación colectiva e individual, fundamentalmente de carácter político frente a un estado nacional.

De acuerdo con Benedicto y Hale (2004), la idea que los pueblos tienen derecho a ser llamados por el nombre que el pueblo usa, y no por el que les da un pueblo ajeno, no ha sido siempre reconocido ni practicado. Por tanto, en lingüística, se ha promovido un esfuerzo por reconocer el nombre que un pueblo usa para denominarse así mismo, como también el nombre que dicho pueblo le da a su lengua. Este derecho es inherente de los pueblos y también es sabida la importancia de la lengua como factor de identificación étnica, siendo la lengua uno de los factores más frecuentes en dicha identificación. En este sentido, Ember \& Ember (2003), plantean que entender el idioma de otro pueblo es esencial para entender la cultura de ese pueblo.

\section{Condición y situación de género}

Según López (1995), comprende como condición de género las característicos que social, cultural e históricamente, son impuestas por la sociedad y que son atribuidas a mujeres, que están llamadas a cumplir con la reproducción biológica y los roles sociales determinados. Estos roles sociales corresponden en su conjunto a las circunstancias, cualidades y características esenciales que define a la mujer como ser social y cultural genérico.

También hace referencia de la situación de mujeres y hombres de acuerdo al nivel de satisfacción de las necesidades. Estas necesidades son alimentación, salud, educación, acceso a servicios básicos, vivienda, etc., que se refleja en todas las estructuras sociales según el ámbito urbano o rural al que pertenezca. También incluye a las nacionalidades, clases, etnias, edades, religiones, ideologías, salud, violencias, arraigo y otros aspectos que definen su situación (López, 1995).

En cuanto a la situación de género, es la forma concreta de cómo se realiza esa condición (formas específicas de subordinación). Muchas veces la subordinación 


\section{GÉNERO E IDENTIDAD}

cambia de forma en diferentes momentos de la historia, pero su esencia significa otras formas de subordinación.

Se refiere a la ubicación que ocupan las mujeres en relación a los hombres y viceversa en la escala social, económica y la esfera política, dando cuenta de las desigualdades entre hombre y mujeres. Para fines de este estudio, la condición y situación de las mujeres serán analizadas tomando en cuenta lo expresado en los párrafos anteriores haciendo énfasis en la condición, es decir la posición de este pueblo en el proceso de demarcación territorial.

\section{Materiales y métodos}

\section{Principio metodológico}

El principio metodológico del presente trabajo de investigación cualitativa se centra en la etnografía. Es decir, la propuesta de investigación nace de la necesidad de las mujeres ramas en que se visibilice su trabajo y sus aportes en el desarrollo con identidad. Para ello, se tomaron en cuenta la isla de Rama Cay y la comunidad de la Zompopera. En este sentido, se puede mencionar que la isla de Rama Cay es la principal comunidad del territorio rama. Así mismo, la comunidad de la Zompopera, se tomó en consideración debido a la experiencia de trabajo con AMIR (Asociación de Mujeres Indígenas Ramas), experiencia que constituye un antecedente importante para la organización de las mujeres ramas.

\section{Actores claves del estudio}

En el presente trabajo se decidió tomar como principales informantes y/o actores claves a los siguientes:

- Profesoras y profesores: por su condición de liderazgo en ambas comunidades, son conocedores de la realidad de la comunidad y principalmente por su rol de formadores.

- Mujeres lideresas y hombre líderes de la comunidad de Rama Cay y la Zompopera.

- Amas de casas que desarrollan actividades económicas y productivas en las comunidades de estudio.

- Mujeres organizadas en AMIR.

88 | CIENCIA E INTERCULTURALIDAd, Volumen 4, Año 2, No. 2, Junio 2009 
- Por tanto, para desarrollar el presente trabajo de investigación etnográfica, los instrumentos y técnicas desarrolladas consistieron en la observación participante, entrevistas, visitas de familiarización y grupos focales.

\section{Fases del estudio}

El proceso de desarrollo en seis fases de la siguiente forma:

Fase l: El diseño de la propuesta de investigación. Esta inició con la elaboración de la propuesta, la cual fue presentada a los líderes comunitarios para su respectiva aprobación. Esta primera fase significó un espacio de familiarización e intercambio con la comunidad y sus líderes alrededor de la propuesta.

Fase II: Se elaboró el protocolo de investigación de acuerdo a lo establecido por la universidad para su respectiva revisión y aprobación por las autoridades de la universidad. Esto incluyó el diseño de los instrumentos de investigación y la identificación de los criterios para la selección de los informantes claves.

Fase III: Se realizó una visita de campo a las comunidades seleccionadas para el estudio de tal forma que pudiera ser validada, revisar y hacer la retroalimentación necesaria a los instrumentos diseñados. Esto acompañado de una guía de observación participante, lo que facilitó el proceso creando un clima de confianza en el desarrollo del trabajo.

Fase IV: Se desarrolló el trabajo de campo, lo cual consistió en la aplicación de los instrumentos mejorados en la fase anterior. Esta fase se ejecutó en dos giras de campo de una semana aproximadamente en ambas comunidades. En este período, además de los grupos focales y las entrevistas, la observación, la permanencia en las comunidades y su cotidianidad permitió profundizar algunos aspectos sobre el aporte de las mujeres ramas en los diferentes ámbitos.

Fase V: Se analizó y procesó la información recolectada para su respectiva presentación y devolución de la información. Esto constituyó un proceso significativo de validación de la información y reflexión colectiva de hombres y mujeres jóvenes y adultos.

Fase VI: Con la validación de la información se procesó y analizó la misma para la elaboración del informe final. 
GÉNERO E IDENTIDAD

\section{Resultados y discusión}

\section{Situación social de las mujeres ramas}

\section{Población y Vivienda de Rama Cay y La Zompopera}

En la comunidad de Rama Cay y la Zompopera de acuerdo con los datos facilitados por el Gobierno territorial del 2008, cuenta con una población de 1018 personas.

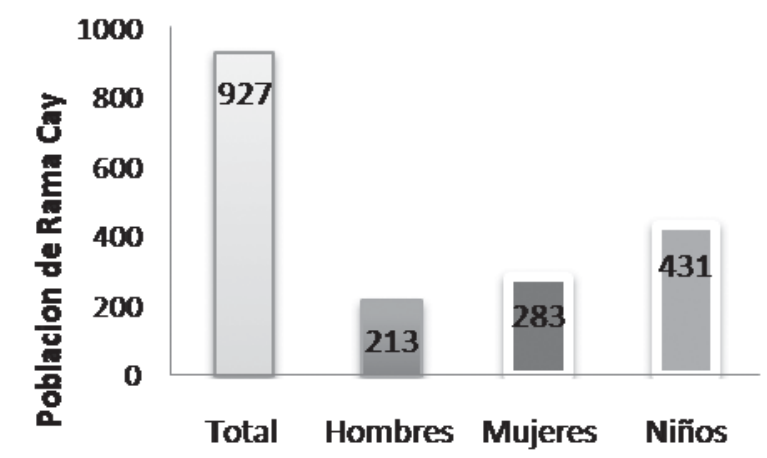

Gráfica 1: Datos demográficos de Rama Cay

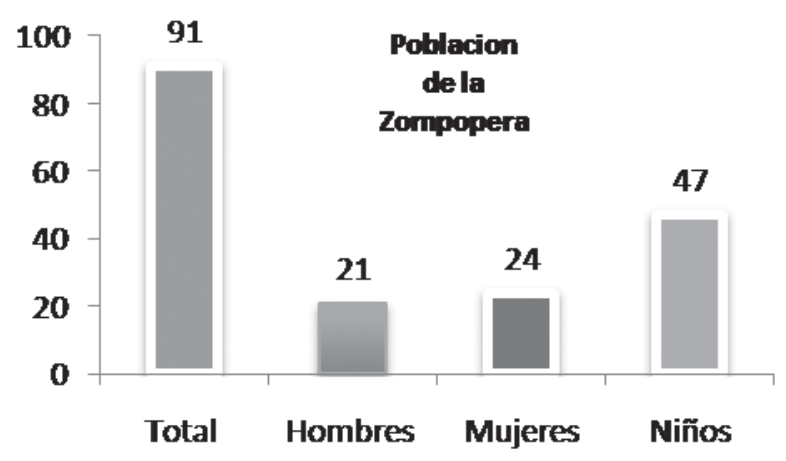

Gráfica 2: Datos demográficos de La Zompopera

Fuente: Censo del Gobierno Territorial Rama 2005-2007.

En la gráfica anterior, se puede observar como la población entre hombres y mujeres, mayoritariamente mujeres en ambas comunidades de estudio. En la comunidad de Rama Cay (Isla) se contabilizan aproximadamente 205 familias quienes habitan

90 | CIenCia e interCulturalidad, Volumen 4, Año 2, No. 2, Junio 2009 
en 185 viviendas, esto nos indica que en cada vivienda habitan un promedio de 5 personas. Sin embargo, es importante mencionar que a pesar de lo anterior, estas viviendas no cuentan con divisiones. Es decir, las viviendas en su mayoría son de madera, con techo de paja y zinc, piso de tierra, lo que hace que los 5 miembros de la familia a pesar de que sean pocos miembros, viven un tipo de hacinamiento. Así mismo, en algunas casas habitan entre 2 a 3 familias, lo que también hace posible que el número de personas que viven en una casa sea arriba de 5 el promedio. Por otro lado, hay que mencionar también que el tamaño de las viviendas es pequeño y esto obedece al poco espacio que queda en la isla para la construcción de casas, siendo muy cercana una de otra.

En las entrevistas realizadas durante el trabajo de campo, se hace mención que en la isla de Rama Cay la mayoría de las familias viven en estado de hacinamiento porque en cada casa habitan de 2 o 3 familias y sus viviendas son muy cercanas unas de otras. En este particular, también manifiestan que es "normal" que las familias vivan cerca unas de otras, sobre todo si los hijos e hijas se casan, estos viven en las casas de sus padres, por lo que lo denominan como "cultura", visto desde las costumbres y patrones de las familias que son heredados de generación en generación. Así mismo, también manifiestan que esta situación se debe a la falta de espacio en la isla en donde ya casi llegan a las 1,000 personas.

Por otro lado, para el pueblo Rama la composición de las familias es extendida, esto significa que se considera "familia" a los padres, a los tíos, a los abuelos, primos, hermanos y parientes cercanos. Esto hace en consecuencia que viva cerca una de otras. También se tiene que mencionar que tanto en la comunidad de Rama Cay como en la Zompopera, el régimen de propiedad es colectivo, lo que hace que todos hagan sus casas y vivan cerca una de otras.

En el caso de la comunidad de la Zompopera el total de familias está en correspondencia con el número de viviendas existentes. Es decir 24 familias en 24 viviendas y con un promedio de 4 personas por vivienda, según los datos del Gobierno territorial Rama. Sin embargo, se pudo constatar que en la comunidad existe un tipo de migración interna intercomunitaria. Es decir, al llegar a las comunidades, muchos de los informantes claves como por ejemplo lideres comunitario, se encontraban en Bluefields, Rama Cay u otra comunidad del territorio Rama. En este particular, se puede mencionar que es poco preciso el censo del gobierno territorial Rama, ya que los líderes comunitarios y algunos miembros de la comunidad plantean que la población promedia está arriba de las 175 personas. 


\section{GÉNERO E IDENTIDAD}

En relación al tipo de vivienda es importante mencionar que por los desastres naturales como huracanes han cambiado la construcción tradicional, en este particular los pobladores de la comunidad de Rama Cay mencionan:

"Antes las cosas eran de palma, sus techos y sus paredes eran de yolillo y el piso era de tierra antes estas casas eran muy frescas y acogedoras, se construían de forma artesanal con la ayuda de amigos vecinos y familia".

Comunitario, Isla Rama Cay, 2008

Hoy en día las casas se construyen de madera y zinc, tratando de que sean más fuertes y resistan a los desastres naturales. Actualmente en la Isla de Rama Cay existe un proyecto de urbanización con la iglesia Evangélica. Este proyecto de urbanización se quiere realizar frente a la Isla de Rama Cay Mane Land (tierra firme) con fondos de cooperación externa de Canadá. Para ello, se han hecho las consultas necesarias a los líderes comunales y el gobierno territorial de tal forma que ya está avalado y la primera etapa beneficiará a 30 familias.

\section{Educación}

Las mujeres ramas ven la importancia de la educación para los pueblos indígenas, esto porque además de las labores comunitarias de liderazgo también en muchos de los hogares son las que enseñan a sus hijos las primeras letras. Así mismo, además de la educación formal, aportan a la educación endógena en donde se transmiten muchos de los conocimientos vinculados a la cosmovisión, valores, creencias y costumbres del pueblo rama. Es decir, la educación endógena corresponde al saber acumulado durante siglos y de las tradiciones ancestrales, el cual asegura la adquisición de los conocimientos y habilidades necesarias para asumir responsabilidades y funciones en la familia y la comunidad, es de esta forma donde se evidencia el aporte al conocimiento y la sabiduría ancestral.

En relación al antes, las mujeres ramas de Rama Cay y la Zompopera, plantean que hace unos años no habían muchas oportunidades para estudiar y la ayuda era limitada. Es decir, antes el acceso a la educación para los hombres y principalmente para las mujeres era casi nulo y que además era monolingüe. Sin embargo, ahora, se cuenta con mejores condiciones para el acceso a la educación, entre estos también se puede mencionar el SEAR - Sistema de Educación Autonómico Regional-, el cual ha permito que hombre y mujeres reciban sus clases en su lengua materna. A pesar de lo anterior, todavía en el caso de las mujeres, estas no logran terminar sus estudios. Según los pobladores de la comunidad mencionan lo siguiente:

92 | CIENCIA E INTERCULTURALIdAd, Volumen 4, Año 2, No. 2, Junio 2009 
La mujer ha sido marginada en estos aspectos de la educación, la mujer tiene problema en la toma de decisiones cuando es una analfabeta, es por esta razón que cuando en una organización hay hombres y mujeres y si la mujer no sabe leer ni escribir esa mujer no toma decisión (...) esta participa de una manera pasiva, en cambio la que mujer tiene educación participa de modo activa, toma decisiones acertadas y no se deja manipular

Líder de la comunidad de Rama Cay, 2008

La cita anterior, plantea que la educación es una herramienta útil para que las mujeres mejoren su situación y condición. Empero, todavía en la cultura rama predomina el rol de la mujer dentro de la casa, al cuidado de los hijos, del marido y la familia. Esto debido a que los hombres a pesar de su bajo nivel académico siempre están en posiciones de liderazgo y de toma de decisión.

En la comunidad de Rama Cay existen dos escuelas, la escuela "Iván Dixon" de Educación Primaria y "Clemente Bayle", escuela secundaria. En la escuela primaria imparten clases de preescolar hasta sexto grado y cuenta con 8 maestros, tres son mujeres y cinco varones. En dicho centro, reciben clases alrededor de 200 niños. Una de las buenas prácticas de la escuela primaria es que los estudiantes reciben clases en idioma rama, las cuales están a cargo del profesor Walter Ortiz originario de Willing Cay, quien tiene 60 años y conserva el idioma nativo de sus padres ramas. El profesor Walter, radica en la isla Rama Cay. En la secundaria laboran 6 profesores, 2 mujeres y 4 varones, hasta este año (2008) en la comunidad de Rama Cay se ha completado la secundaria, es decir, hasta quinto año de bachillerato. Por tanto, para este año, se espera poder realizar la primera promoción de bachilleres en la isla.

La idea de que se tenga ya los primeros bachilleres es un logro importante para la educación de los jóvenes de la comunidad y principalmente para las mujeres. Es decir, el hecho de que se tenga acceso a un bachillerato en la isla, pone en disposición positiva a las jóvenes, quienes en su mayoría inician su maternidad a muy temprana edad.

En la comunidad de Zompopera no existe en términos de infraestructura una escuela; sin embargo, en la casa comunal la niñez recibe clases. Para ello, cuentan con una maestra que atiende a 40 niñas y niños de diferentes grados de primero a quinto grado (esta profesora da clase de multigrado). La situación en la comunidad de la Zompopera es limitada por el hecho de contar solamente con una profesora que atiende a 40 estudiantes en un mismo salón; pero también es importante mencionar que cuando llueve y el río crece, las niñas y niños que viven al otro lado del río de la 


\section{GÉNERO E IDENTIDAD}

comunidad no pueden cruzar para recibir sus clases. Ante esto, también se podría señalar como una Buena Práctica (como aporte de la mujer) el hecho de que para esa niñez no pierdan sus clases los deja viviendo en su casa mientras el río baja su nivel, la maestra se encarga de su cuido y su alimentación, lo cual va por su cuenta propia.

\section{Salud Comunitaria}

La salud para la mujer Rama, es vista desde el bienestar de la familia. Es decir, el gozar de buena salud sin enfermedades, principalmente las hijas e hijos sin calenturas, diarreas u otros malestares.

La isla de Rama Cay cuenta con un centro de salud que se abastece del suministro que se recibe de Bluefields. En el caso de la Zompopera, sus pobladores no cuentan con médicos, enfermeras ni puesto médico, esta comunidad de alguna forma u otra es atendida por brigadistas de salud que son enviados esporádicamente por el MINSA.

Por otro lado, la población de Rama Cay crece cada vez más sin mayores controles y es evidente ver en el gráfica 2 un acelerado crecimiento de niñas y niños en la comunidad. En consecuencia, este crecimiento poblacional conlleva a problemas sociales y mayor necesidad de medicinas y atención en salud. En relación al alto índice de natalidad se puede suponer que esto se debe a cuestiones culturales, acompañada de la falta de una adecuada educación sexual reproductiva de acuerdo a sus normas y tradiciones.

En relación a la salud, tanto en las comunidades de Rama Cay como la Zompopera, estas no cuentan con ningún tipo de servicios básicos, principalmente agua potable. El agua de consumo en ambas comunidades proviene de los pozos y agua de lluvia tratadas con cloro y hervida. En términos generales, existe un déficit de servicios básicos, ya que el 100\% no cuenta con agua potable, energía eléctrica, transporte colectivo regular entre otros. Así mismo, la situación del agua, también ha provocado que las enfermedades más comunes sean la diarrea, gripe y tos. En relación a estas enfermedades, los ramas acuden a la medicina occidental, pero a la vez hacen uso de la medicina tradicional. Esto, desde luego, responde a sus costumbres y tradiciones, pero también a la falta de medicinas en el puesto de salud.

En Rama Cay y la Zompopera, paralelo al sistema de atención del MINSA también están los agentes tradicionales de salud, quienes son las parteras y curanderos. En el caso de las parteras es una práctica muy común el uso de sus servicios en la comunidad. Por ejemplo, si una mujer requiere de sus servicios paga un monto de $C \$ 200$ córdobas por 8 días. En estos días, la partera cuida al bebé y a la madre. 
Trabajo de las parteras:

- Lava la ropa.

- Cuida al bebé recién nacido y a la madre.

- La partera le facilita medicina tradicional que le ayuda a limpiar el útero y la matriz, esta contiene manzanilla y hombre grande.

Además de los agentes tradicionales de salud, también existe un grupo de brigadista de salud que se encarga de realizar jornadas de vacunación, abatizaje de las aguas e imparten charlas sobre la higiene, enfermedades de trasmisión sexual y el cuido al medio ambiente.

En la comunidad de la Zompopera, como se menciona anteriormente no cuenta con puesto de salud, por lo que en ocasiones brigadistas de salud atienen la comunidad. Así mismo, recientemente, los brigadistas solamente han facilitado el suministro de tratamientos, los cuales son entregados al responsable de la casa comunal quien entrega los analgésicos que se creen conveniente, en caso de gravedad lo tienen que llevar hasta la Isla de Rama Cay para ser atendido por el médico o en última instancia trasladarlo a Bluefields (dependiendo de la gravedad del paciente).

Las personas de la Zompopera también practican la medicina natural porque dicen que les da buen resultado y no tienen mayor costo puesto que las plantas están en los patios de sus casas o en los ríos o montes cercanos a su casa de habitación. En este particular, es importante mencionar que es la mujer quien cuida el patio, así también la que se encarga del manejo de las plantas medicinales, lo que desde luego representa un aporte significativo a la salud desde el trabajo de las mujeres.

\section{Participación de la Mujer Rama}

Históricamente las mujeres han desempeñado un triple rol ante la sociedad. Estos roles se han determinado por medio de la participación, tanto en funciones productivas, reproductivas y así también de gestión comunal, con el fin de visualizar la carga laboral de ellas.

Se define las funciones productivas a toda tarea que contribuya económicamente al hogar y a la comunidad. Asimismo las funciones o actividades reproductivas corresponde al conjunto de actividades que comprenden el cuido y el mantenimiento del hogar, gestar, dar a luz, criar y educar a los hijos, atención a la salud, preparación de los alimentos, recolección de agua, leña, compra de provisiones, los quehaceres domésticos y el cuido de la familia: esta actividades lamentablemente no están categorizadas como 


\section{GÉNERO E IDENTIDAD}

económicos por no obtener una compensación monetaria. En el ámbito productivo, las mujeres ramas de la Isla de Rama y la Zompopera, han aportado a las prácticas económicas de patio (ver cuadro anexo).

Nosotras las mujeres de la comunidad de Rama Cay y Zompopera ayudamos a nuestros compañeros, hombre o esposos en el monte, criamos gallinas, cerdos, sembrando maíz, banano, yuca, ambos recolectamos las cosechas, aquí toda la familia nos dedicamos al cultivo de la tierra.

Mujer Rama, 2008.

Es decir, las mujeres Ramas, además de apoyar las labores del campo, tienen la responsabilidad de cuidar a las niñas y niños, preparar la comida, lavar y cuidar a los animales domésticos en el caso de que tengan. En la Zompopera, las mujeres ramas manifiestan su cotidianidad de la siguiente forma:

Aquí en la Zompopera las mujeres nos levantamos muy temprano, preparamos el desayuno y el hombre, y luego nos vamos al campo a ayudar en la recolección o siembra, pero nos venimos un poco antes a la casa para hacer el almuerzo, así cuando el hombre viene del campo sólo viene a comer, yo creo que nosotras las mujeres trabajamos más que ellos porque picamos la leña, jalamos agua, lavamos, cocinamos y cuidamos a los niños y les ayudamos en el campo.

Mujer Rama de la Zompopera, 2008.

Según las mujeres de la Zompopera, tienen la iniciativa de organizarse, por lo que apenas hace unos meses han estado tratando de conformar una organización de Mujeres. Esto con el propósito de velar por los intereses de las mujeres y realizar proyectos que vayan en beneficio de las mismas.

\section{Actividades económicas, familiares y comunitarias de las mujeres indígenas ramas}

\section{Actividades económicas provenientes de la pesca}

Una de las principales actividades económicas de las familias ramas corresponde a la comercialización de la pesca. El precio de estos, corresponde al lugar de venta así como también a la temporada. 
Cuadro 1: Principales productos del mar que comercializa la comunidad de Rama Cay y La Zompopera.

\begin{tabular}{cccccc}
\hline Producto & Ostiones & Almejas & Chacalines & Pescado & Camarones \\
\hline $\begin{array}{l}\text {-Rama Cay } \\
\text {-Zompopera }\end{array}$ & C\$ 60 libra & C\$15 Libra & C\$15 Libra & C\$15 Libra & C\$ 30 Libra \\
\hline Bluefields & C\$ 80 libra & C\$25 Libra & C\$25 Libra & C\$19 Libra & C\$ 45 Libra \\
\hline Fuente: Elaboración propia & & & &
\end{tabular}

En el cuadro 1, se presentan los precios promedios de la comercialización de los productos de la pesca. La comercialización de estos productos, está en función de la temporada, es decir, en los meses de febrero y abril se encuentran chacalines; en julio, agosto, septiembre y noviembre se pesca camarones, por lo que la comercialización de camarones es importante para la economía familiar-comunitaria, principalmente porque es una de las temporadas que abarca la gran mayoría del año. Así mismo, los ostiones y almejas que prácticamente todo el año se puede contar con este producto. Es importante mencionar que estos dos últimos productos son de los mayores aportes económicos que realizan las mujeres a la economía familiar ya que están en casi todos los meses de año.

\section{Actividades económicas provenientes de la caza y la agricultura}

En relación a la caza de animales, esta se ha considerado como una práctica muy común entre las comunidades indígenas ramas de Rama Cay y la Zompopera. Los comunitarios, salen a cazar casi semanalmente. Actualmente, muchas personas practican la caza con escopeta, pero antes usaban sólo la lanza. Para la caza con lanza, los hombres usaban achote para salir en grupos de 2 ó 3 personas. En estas actividades, las mujeres también cazaban acompañando a sus parejas. Así mismo, en las comunidades de Rama Cay y la Zompopera, se cazan chanchos de monte (jabalí), iguana, venado, pero desde luego, el número de animales ha ido disminuyendo con el pasar del tiempo.

En relación a la agricultura, esta se hace a orillas de los ríos de Dokuno y Torsuani siempre dentro del territorio Rama. Sin embargo, estos hacen rotación de cultivos y después dejan descansar la tierra. Según el orden, los comunitarios plantearon que en las áreas de cultivo, después de los granos básicos, cultivan banano y dejan descansar la tierra para que no se agote.

En el cuadro 2, se presentan los precios promedio de los productos que se comercializan entre Bluefields, Rama Cay y la Zompopera. Es importante mencionar que 


\section{GÉNERO E IDENTIDAD}

en los meses de marzo a diciembre se cosecha yuca, malanga. Así mismo, todo el año se puede encontrar plátano y quequisque.

Cuadro 2: Principales productos de la agricultura que se comercializa en las comunidades de Rama Cay y La Zompopera.

\begin{tabular}{lll}
\hline \multicolumn{1}{c}{ Productos } & \multicolumn{1}{c}{$\begin{array}{c}\text { Comunidad de Rama } \\
\text { Cay y la Zompopera }\end{array}$} & Bluefields \\
\hline Yuca & $\mathrm{C} \$ 3 \mathrm{lb}$ & $\mathrm{C} \$ 5 \mathrm{lb}$ \\
\hline Malanga & $\mathrm{C} \$ 3 \mathrm{lb}$ & $\mathrm{C} \$ 8 \mathrm{lb}$ \\
\hline Quequisque & $\mathrm{C} \$ 6 \mathrm{lb}$ & $\mathrm{C} \$ 8 \mathrm{lb}$ \\
\hline Maiz & $\mathrm{C} \$ 4 \mathrm{lb}$ & $\mathrm{C} \$ 5 \mathrm{lb}$ \\
\hline Plátano & $\mathrm{C} \$ 3 \mathrm{Unidad}$ & $\mathrm{C} \$ 5 \mathrm{lnidad}$ \\
\hline Banano & $\mathrm{C} \$ 1$ Unidad & $\mathrm{C}$ 2 lb \\
\hline Carbón & $\mathrm{C} \$ 45$ saco & $\mathrm{C} \$ 60$ saco \\
\hline Leña & $\mathrm{C} \$ 40$ el rollo & $\mathrm{C} \$ 60$ el rollo \\
\hline
\end{tabular}

Fuente: Elaboración propia

Es importante mencionar que para la comercialización de los productos que se presentan en el cuadro 2, la mujer participa activamente en dicho proceso. Razón por la cual siempre la podemos encontrar acompañando a sus maridos en Bluefields y en Rama Cay, ellas los venden desde su casa.

\section{Aporte de la mujer rama a la revitalización cultural}

Los rama son uno de los Pueblos Indígenas en Nicaragua, cuya población e idioma dramáticamente se ha ido extinguiendo a lo largo de los años. En la actualidad apenas existen unos diez ancianos hablantes de lengua rama y una población bastante pequeña.

\section{Identidad de la mujer y del pueblo rama}

Para la mujer y el pueblo rama en su totalidad, la identidad está ligada a dos elementos importantes:

- La Lengua (Idioma rama, especialmente el rama-creole).

- El nacimiento de la persona en las comunidades del territorio rama y de padres ramas. 
En relación a la primera característica sobre el idioma rama, es bien conocida la casi desaparición del idioma. Sin embargo, a pesar de ello, en las comunidades de Rama Cay y la Zompopera, se habla el "rama-creole". El rama-creole, es un idioma en donde se mezclan elementos lingüísticos de ambos idiomas, dando como resultado una particular pronunciación en la comunicación.

Lo anterior, coincide con los planteamientos de Rizo, Benedicto y Hale establecidos en el marco teórico, en donde se menciona que la identidad de los pueblos indígenas y comunidades étnicas de la Costa Caribe nicaragüense tienen diferentes grados de mestizajes, por lo que la identidad étnica es el resultado de una auto adscripción o representación colectiva e individual. Por tanto, en el caso del pueblo rama, el hecho de denominar su idioma como rama-creole lo constituye como unos de los principales elementos de la identidad del pueblo, constituyendo la lengua factor de identificación étnica.

La lengua rama, según los jóvenes, es algo que sólo los ancianos hablan. Algunos de ellos, mencionan que esto se debe a que los mayores ${ }^{[18]}$ cuando les hablaban a los jóvenes lo hacían mezclando el rama-creole, por lo que en la actualidad dicen en Bluefields, que la gente de Rama Cay hablan un rama-creole ${ }^{[19]}$.

La casi desaparición de la lengua rama se debe, principalmente a dos factores convergentes, los cuales han hecho que se abandone la lengua rama como principal medio de comunicación en la isla, siendo reemplazado por el inglés criollo. El primero de estos factores es la proximidad de la isla a la ciudad de Bluefields y por ende el contacto cultural con los creoles. Por otro lado, la llegada de la misión morava en la segunda mitad del siglo XIX, en donde fundaron la iglesia y la escuela,

Por otro lado, otro elemento importante de identidad se identifica a partir del nacimiento de una mujer y un hombre rama. Así mismo, algunos miembros de la comunidad establecen como elementos de identidad: "Su forma de vestir, de caminar, con su cabello largo liso, andar descalzo", rasgos que actualmente siguen siendo válidos para los que viven en las comunidades.

[18] Padres de familia, abuelos, tíos, personas ancianas.

[19] Rama - Creole es una mezcla del creole de Bluefields, con el Miskito y la lengua Rama, algunos dicen que hasta una pequeña mezcla del Alemán, por eso su pronunciación es diferente al Creole hablado en Bluefields. 


\section{GÉNERO E IDENTIDAD}

En las entrevistas realizadas, se planteaba como un elemento de la identidad el entorno. Esto visto como el territorio. Así mismo, sus valores y nivel de compromiso con los recursos naturales, donde la tierra es vista como la madre de todos.

“También el pueblo rama se identifica más con la naturaleza porque es el único medio de sobrevivir, allí es donde tiene la comida, están los recursos hídricos, la forma de tierra y la lengua misma que está en peligro de desaparecer".

\section{Costumbres y Tradiciones}

La población de la comunidad indígena rama tiene sus propias costumbres y tradiciones, entre estas todavía se hacen cayucos y velas que es el único medio de transporte. También se hacen lanzas de hierro para cazar animales del monte, hacer guarapo con la caña cuando no hay azúcar esto es para endulzar el té.

El pueblo rama tiene muchos platos típicos para los cuales usan ingredientes propios de la Costa. Entre estos podemos mencionar: el cacao con chile, pescado, coco, pejibayes, pinol de almendro, tamal de almendro, queque de almendro con banano maduro, banano maduro asado, banano verde (8 días en agua, le echan sal y lo pone a ahumar), plátano maduro seco fermentado, carne de chancho de monte (hacen un hoyo en la tierra, ponen palos, carbón, leña encendida, hojas húmedas, bijagua, carne fresca y hojas encima).

También los adultos tienen creencias en los fenómenos naturales, una de las costumbres y tradicionales que no conservan actualmente el "Shauda". Es decir, la festividad del Shauda es recordado con alegría por los comunitarios de mayor edad en la Isla. Primeramente porque para ellos era un momento en donde las familias que habitaban la Isla podían unirse para celebrar el triunfo de una persona al momento de atrapar un manatí, pues en esos tiempos pescar un animal de ese volumen era un logro significativo.

El Shauda sólo se celebraba cuando un pescador lograba atrapar un manatí, porque que la carne del Manatí daba abasto para alimentar a toda la población que en aquel entonces vivía en la Isla de Rama Cay. Los hombres al escuchar sonar el cuerno de la vaca, sabían inmediatamente que en la isla habría Shauda, entonces las mujeres se preparaban para cocinar la sopa con carne de manatí y dar de comer a todos los habitantes de la isla. 
El señor Juan Daniels, dice recordar las técnicas al momento de cocinar el manatí, las mujeres cocinaban la carne de manatí. Un aspecto importante era al momento de servir la carne, puesto a que el pescador que atrapó el animal marino no podía comer de la parte de la costilla. Esto debido a que le traería mala suerte y nunca más podría atrapar otro manatí, de igual forma los perros no podían lamer los huesos del manatí porque también traería mala suerte al pescador, por lo que las mujeres eran las encargadas de echar en sacos los huesos y tirarlos en los Cayos de Walker Cay y Pigeon Cay ${ }^{[20]}$ que se localizan frente a la isla. La mujer aseguraba que el hombre tuviera la oportunidad de volver a atrapar un manatí y lo mismo cuidar a los perros (cuidar la suerte de la familia).

\section{El aporte de miss Nora Rigby a la lengua rama}

Eleanora Rigby conocida como miss Nora era originaria de la comunidad de Rama Cay. Ella era hija y esposa de hablantes nativos del idioma rama. Miss Nora era la mejor hablante de la lengua rama y pasaba el mayor tiempo en la comunidad de Cane Creek.

En 1985 miss Nora empezó a compartir sus conocimientos sobre el idioma rama con los lingüistas que visitaban la comunidad de Rama Cay. Posteriormente, a partir de 1987, miss Nora empezó a ir diariamente a la escuela de Rama Cay para enseñar la lengua rama a los niños de pre-escolar con la participación de los maestros de la misma escuela.

Su labor docente y el rescate de la lengua Rama fue realizada durante aproximadamente 10 años; en 1997 fue su último año de facilitar clases en idioma rama. Su labor es una de las más reconocidas en Nicaragua, debido a que aportó significativamente a la construcción de un Diccionario Rama y de muchos artículos sobre el idioma rama.

Así mismo, gracias a la labor de miss Nora, actualmente en la escuela de Educación Primaria de Rama Cay, se facilitan clases en idioma rama. Por tanto, el aporte de miss Nora como mujer rama es una de las prácticas de revitalización y rescate cultural más importante para el pueblo rama.

[20] Rama Cay, Walker Cay y Pigeon Cay son uno de los ocho Islotes que conforma el archipiélago en la Bahía de Bluefields. 


\section{GÉNERO E IDENTIDAD}

\section{Conclusiones}

- La situación social de las mujeres ramas en los últimos años ha mejorado en relación al liderazgo comunitario. Así mismo, se visualizan aportes cualitativos en los roles productivos, reproductivos y comunitarios de la mujeres ramas.

- La mujer rama aporta significativamente a la economía familiar con la venta de los productos provenientes de la pesca y la agricultura. Así mismo, apoya al trabajo de los hombres en las labores agrícolas y comercialización de los productos. De igual manera, en las actividades económicas de subsistencias en la comunidad (pulperías, profesoras, maestras, etc.).

- La mujer rama es el pilar de la educación informal, se encarga de velar por la educación en los hijos. De igual manera, se encarga de transmitir las costumbres y tradiciones.

- La participación de la mujer rama es funcional por cuanto están organizadas en AMIR para la realización de tareas específicas, pero también en otros grupos comunitarios.

\section{Lista de referencias}

Benedicto, H., Hale, K. (2004). ¿Sumo, mayagna, Tuahka, Panamahka, Ulwa? Lengua e Identidad Étnica. Revista WANI 38 (6-24).

Cárdenas, Víctor Hugo (1997 ). Los Pueblos Indígenas, el Desarrollo y la Democracia en América Latina. Conferencia dictada el 4 de febrero en el BID, Washington D.C.

Cunningham, M. (1999). Memoria del Primer Seminario Internacional de Biodiversidad, Propiedad Intelectual y Derecho indígena. Siuna, Nicaragua.

Ember, C., Ember, M. (2003). Antropología Cultural. Madrid, España. Prentice Hall.

Guglielmi, G, (2006). Las comunidades indígenas y étnicas de Pear Lagonn: Entre la modernidad y tradición. Revista WANI 44 (37-45).

Hernández, Tanaka, N., , I. (2004). Conflicto por el uso de la tierra y otros Recursos Naturales entre los colonos Mestizos de la Frontera Agrícola en Monte Creek y los Indígenas Ramas de la comunidad de Punta de Águila. Monografía. URACCAN-Bluefields

López, A. (1995). Cuerpo Humano e Ideología. UNAM, México. 
Moreno, J. et al. (2000). Ansiedades del pueblo Rama. Revista Universitaria Caribe 2(131-146).

Muller, G. (2003). El territorio Rama y Creole en el contexto de la nueva ley de demarcación. Revista WANI. Nicaragua. 34 (6-19).

PNUD (2005) Informe de Desarrollo Humano de la Costa Caribe Nicaragüense -IDHCC-.

Obando V. et al. (1999). Revitalización cultural del pueblo Garífuna de la Costa Caribe Nicaragüense. IPILC-URACCAN.

Rizo, M. (2003). Cultura. IPILC-URACCAN.

Sambola, K. (2004). Análisis de género del proceso de demarcación territorial, en las comunidades Garífunas del bloque Multiétnico de Laguna de Perlas, RAAS en el período 2003. Monografía. URACCAN-Bluefields. 


\section{Anexos}

\section{Anexo 1. Fotos del trabajo de campo de la investigación} en las comunidades de Rama Cay y la Zompopera.

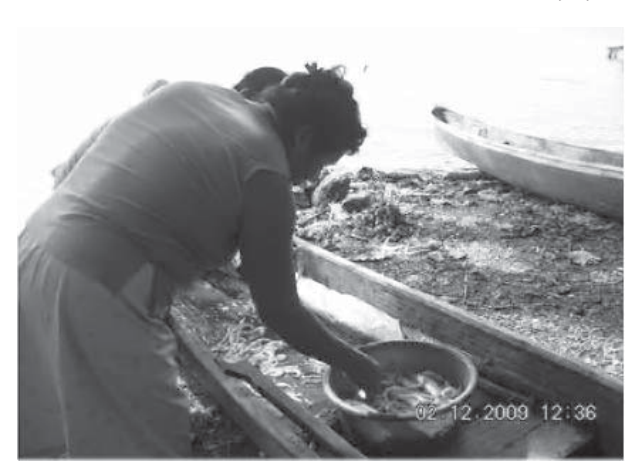

Foto 1: $\quad$ Mujer Rama de Rama Cay en Labores de la Pesca.

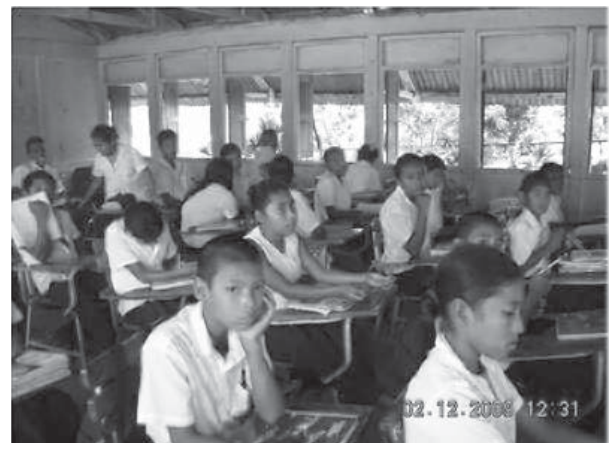

Foto 3: Escuela primaria de Rama Cay.

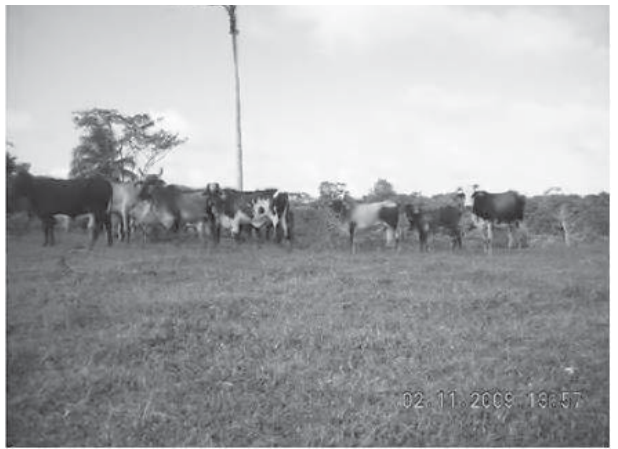

Foto 4: $\quad$ Finca de AMIR La Zompopera

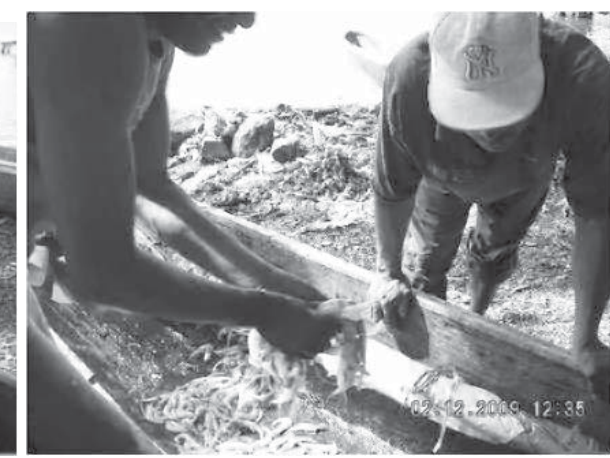

Foto 2: $\quad$ Hombre en labores de la Pesca en la comunidad de Rama Cay.

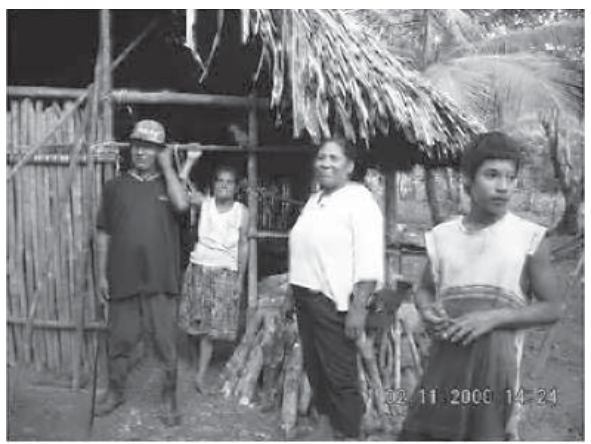

Foto 4: $\quad$ Comunidad La Zompopera.

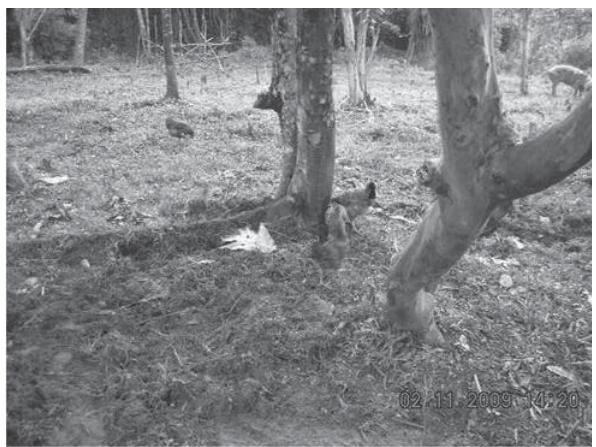

Foto 5: Comunidad La Zompopera. 


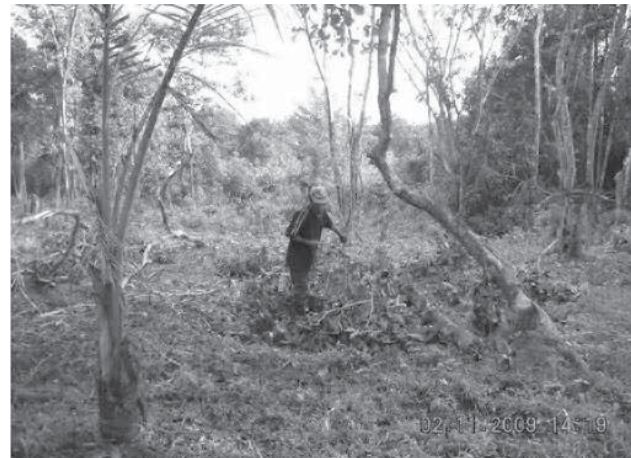

Foto 6: $\quad$ Producto de la comunidad de La Zompopera

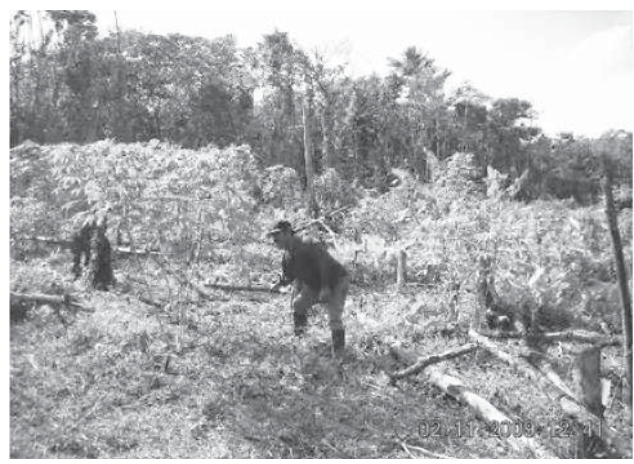

Foto 8: $\quad$ Plantaciones en la Comunidad de la Zompopera.

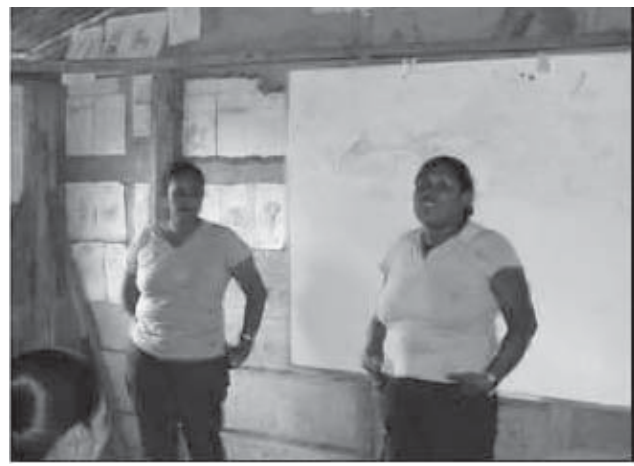

Foto 10: Taller de Validación en la Comunidad de Rama Cay

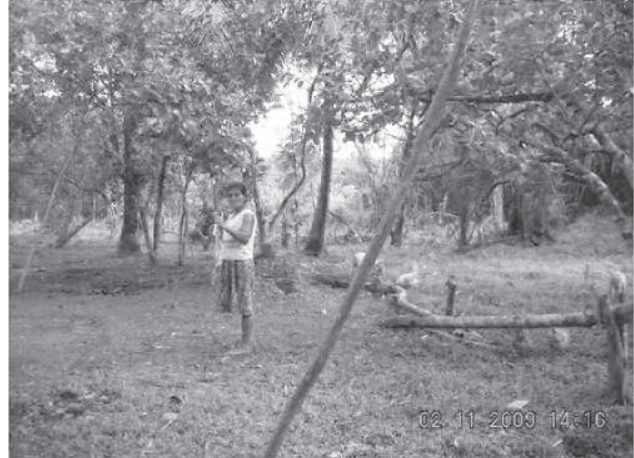

Foto 7: Comunidad de la Zompopera

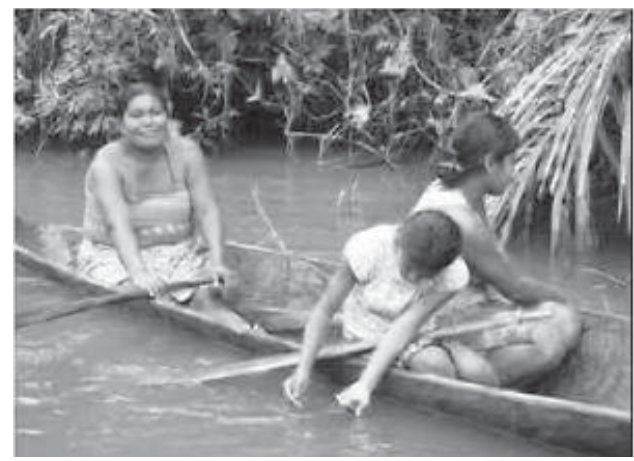

Foto 9: $\quad$ Mujeres ramas en el río

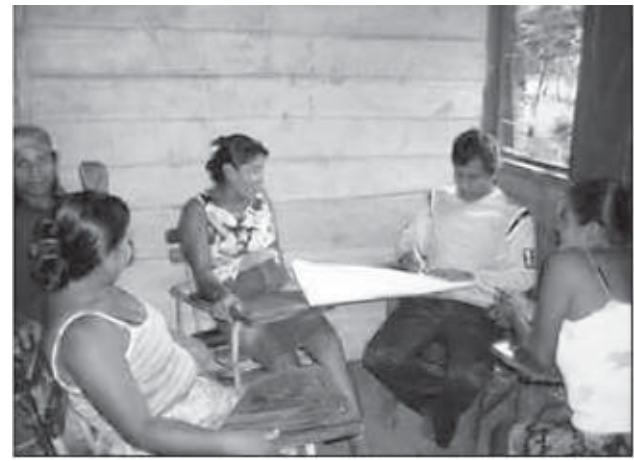

Foto 11: Taller de Validación comunidad La Zompopera. 